\title{
Une gestion responsable des expositions temporaires à la Bibliothèque nationale de France
}

Accountable management of temporary exhibitions in the French National Library

Serge Derouault et Anne-Hélène Rigogne

\section{(2) OpenEdition}

Journals

Édition électronique

URL : http://journals.openedition.org/ocim/1035

DOI : 10.4000/ocim.1035

ISSN : 2108-646X

Éditeur

OCIM

Édition imprimée

Pagination : 10-15

ISSN : 0994-1908

Référence électronique

Serge Derouault et Anne-Hélène Rigogne, « Une gestion responsable des expositions temporaires à la Bibliothèque nationale de France », La Lettre de I'OCIM [En ligne], 140 | 2012, mis en ligne le 01 mars 2014, consulté le 01 mai 2019. URL : http://journals.openedition.org/ocim/1035 ; DOI : 10.4000/ ocim. 1035 


\title{
Une gestion responsable des expositions temporaires à la Bibliothèque nationale de France
}

\author{
Serge Derouault et Anne-Hélène Rigogne *
}

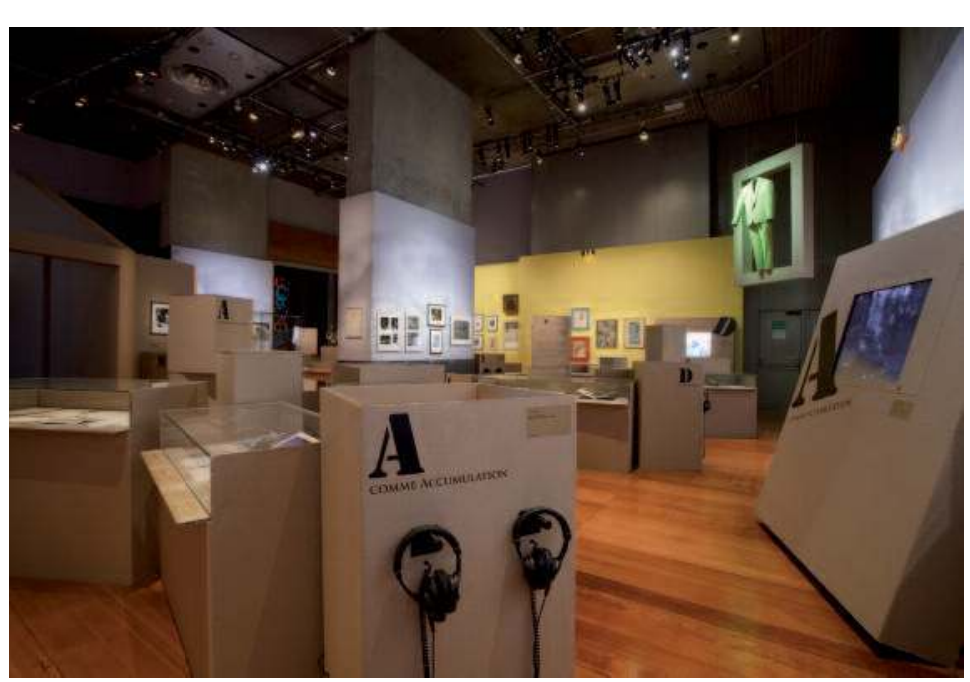

Une scénographie en carton pour l'exposition lonesco en 2009 (scénographie : Alain Batifoulier)
@ BnF/David-Paul Carr

* Serge Derouault est responsable technique du service des expositions de la $\mathrm{BnF}$ serge.derouault@bnf.fr Anne-Hélène Rigogne est conservateur en chef des bibliothèques, adjointe du chef du service des expositions de la $\mathrm{BnF}$ anne-helene.rigogne@bnf.fr
Depuis quelques années, le service des expositions de la Bibliothèque nationale de France propose des expositions plus respectueuses de l'environnement, tout en tenant compte des exigences esthétiques, et des impératifs budgétaires et juridiques : après avoir rappelé quelques éléments du contexte, les responsables de ce service tracent à grands traits les pistes explorées, savants compromis entre aspirations généreuses et réalités.

\section{Présenter des expositions temporaires dans une grande bibliothèque}

Dès 1994, à la faveur de l'ouverture du site François Mitterrand, la Bibliothèque nationale de France ${ }^{(1)}$, s'est lancée dans une ambitieuse politique d'action culturelle et a vu le nombre de ses expositions programmées annuellement croître de manière considérable. Valoriser les collections de la Bibliothèque par des expositions a toujours fait partie de ses activités traditionnelles, depuis l'an II... mais cette mission fondamentale, inscrite dans le décret du 3 janvier 1994 instituant le nouvel établissement public, est dorénavant confiée à un service de 20 personnes (chef de service, chargés d'exposition, techniciens, administratifs) qui produit environ 17 expositions de tailles diverses (100 à $800 \mathrm{~m}^{2}$ ) par an sur différents sites : historiques, comme ceux de Richelieu, de la Bibliothèque de l'Arsenal et de la Bibliothèque-Musée de l'Opéra, ou récents comme le site François-Mitterrand dont les trois galeries sont très bien équipées techniquement. 
La typologie variée des expositions de la BnF est liée à l'encyclopédisme de ses collections, et aux enjeux de son actualité. Leur programmation est parfois dictée par des dons ou des acquisitions comme les expositions Markus Raetz et Casanova, la passion de la liberté. Elles peuvent traiter d'un sujet monographique comme l'œuvre d'un écrivain ou d'un photographe : Depardon, Boris Vian. Elles peuvent aussi s'intéresser à une figure mythique de la littérature médiévale comme La Légende du roi Arthur ou ressortir à l'histoire des idées ou des mentalités : Lumières, Utopies. Certaines collections spécifiques sont présentées et mises en perpective : La Bastille ou l'enfer des vivants, sur les archives de la célèbre prison, Miniatures flamandes, sur les manuscrits enluminés commandés par les ducs de Bourgogne. Les documents exposés sont de tous types : manuscrits enluminés, manuscrits contemporains, imprimés, estampes, photographies, cartes, globes, monnaies et médailles, costumes, audiovisuel, et documents numériques...

Le service des expositions ne bénéficie pas de l'infrastructure d'un musée et la plupart des prestations, comme la conception scénographique, le graphisme, les travaux d'aménagement sont externalisées. Toutefois, le service dispose en interne d'un parc de vitrines permanent, d'équipements multimédias et audiovisuels et les prestations audiovisuelles et d'éclairage sont réalisées par nos techniciens.

\section{Intégrer le développement durable à nos pratiques : une démarche progressive mais volontariste}

En 2007, suite au Grenelle de l'Environnement, la BnF s'est engagée dans une démarche volontariste, impulsée par la directrice générale adjointe de l'époque, Valérie Vesque-Jeancard. Un bilan carbone a été réalisé en 2008 ; il a été suivi d'un audit thermique du bâtiment, d'une sensibilisation du personnel, d'achats de consommables responsables... Le parc d'imprimantes a été diminué et actuellement, $16 \%$ des marchés BnF intègrent des éléments liés au développement durable. Les efforts se poursuivent.

Le service des expositions s'est bien sûr trouvé engagé dans ce mouvement général, et dès 2007, nous avons fait des essais timides sur l'exposition Zao Wu $K i$, en incluant dans le cahier des charges quelques recommandations sur la labellisation du bois et de la peinture. Bien que cette démarche soit loin de faire l'unanimité dans le service, nous avons demandé à bénéficier d'une étude sur nos pratiques pour être plus pertinents dans nos tentatives.
La direction a financé une étude de la société Atémia ${ }^{(2)}$, trouvant dans les expositions un terrain d'expérimentation identifiable et visible sur le court terme. Cette étude a eu lieu de mars à juin 2008, elle s'est déroulée en trois temps : bilan de l'existant (entretiens, études de nos documents administratifs et financiers, entretiens avec nos prestataires et visites de terrain), analyse de l'impact environnemental de nos actions et enfin, recommandations avec en sus la remise d'un guide pour nos interlocuteurs, scénographes et graphistes (3).

Cette étude a servi de formation et dès 2008, nous avons essayé d'appliquer les recommandations d'Atémia. Un des points importants de cette étude, est qu'elle a été menée avec des agents de différentes directions de la BnF et qu'elle a permis de constituer un réseau interne sensibilisé à ces questions. Le développement durable étant une problématique plus culturelle que technique, il était essentiel d'avoir pu dialoguer avec les responsables du laboratoire de conservation et d'avoir leur aval pour notre démarche ; de même, les cadres administratifs et financiers présents ont pu nous aider à avancer dans la rédaction de nos futurs marchés.

Cette étude a servi de déclencheur et à sa suite des procédures et des actions, sur lesquelles nous reviendrons, ont été mises en place. En 2010, un travail de refonte du guide à l'intention des scénographes a été initié avec Atémia, et une formation pour six agents du service avec mise en place d'un outil d'évaluation a été organisée. En 2011, nous nous sommes lancés dans l'évaluation de nos expositions du point de vue du développement durable.

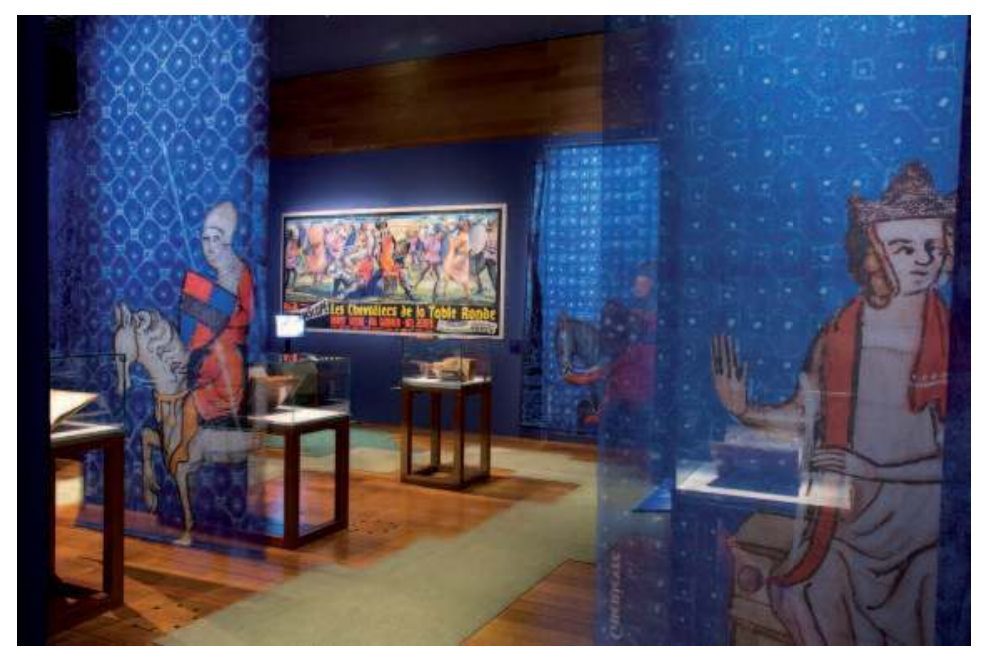

Les voilages de l'exposition La légende du roi Arthur en 2009

(scénographie : MAW) () BnF/Pascal Lafay 


\section{La scénographie, un impact essentiel sur le projet}

La scénographie détermine l'ensemble des matériaux utilisés sur le projet. Le stade de la conception est essentiel, aussi à chaque concours, en sus du cahier des charges qui précise déjà notre sensibilité aux objectifs du développement durable, et de la liste des pièces, nous donnons aux équipes en compétition notre « guide de recommandations à l'attention des scénographes et graphistes ». Celui-ci contient des prescriptions méthodologiques et des fiches pratiques sur les labels et matériaux.

Des exigences en matière de développement durable sont intégrées dans nos marchés de scénographie tandis que les esquisses et les notes d'intention remises sont examinées en fonction de ce critère, s'il n'est pas en contradiction avec la qualité esthétique et la cohérence du projet. Nous cherchons bien sûr à favoriser les projets limitant la matière utilisée, jouant sur les hauteurs, encourageant les formes économes et utilisant les matériaux recyclés ou recyclables.

Ainsi, les cloisonnements en carton alvéolaire de l'exposition Boris Vian (2011, scénographie : Je formule), la scénographie en carton de l'exposition Ionesco (2009, scénographie : A. Batifoulier) et les voilages de l'exposition La légende du roi Arthur (2009, scénographie : MAW), repris dans l'exposition Qumran (2010, scénographie : MAW) ont été particulièrement appréciés. L'obligation d'utiliser au maximum le parc permanent de vitrines faisait partie des cahiers des charges depuis 1996. Désormais nous allons plus loin : les contrats des scénographes incluent la possibilité de réutiliser une partie des matériaux de leur scénographie pour l'exposition suivante, et à chaque mise en concurrence, nous livrons le plan de l'exposition précédente et organisons une visite sur place. Le plancher de l'exposition Raymond Depardon (2010, scénographie : Olivia Berthon, Julia Kravtsova) a ainsi été réutilisé dans l'exposition Richard Prince, American Prayer (2010 scénographie: D. Adjaye).

Un certain nombre de contraintes doivent être intégrées par les scénographes aux documents de consultation des entreprises (DCE) et respectées par nos prestataires. Pour les travaux d'aménagement, le label PEFC (Plan European Forest Certification) ou l'écolabel FSC (Forest Stewardship Council) pour les bois massifs sont exigés. Nous utilisons quelquefois l'aggloméré ou du médium qui ne sont pas totalement satisfaisants : nous n'avons pas encore passé le cap de l'obligation d'utilisation de nouveaux matériaux type

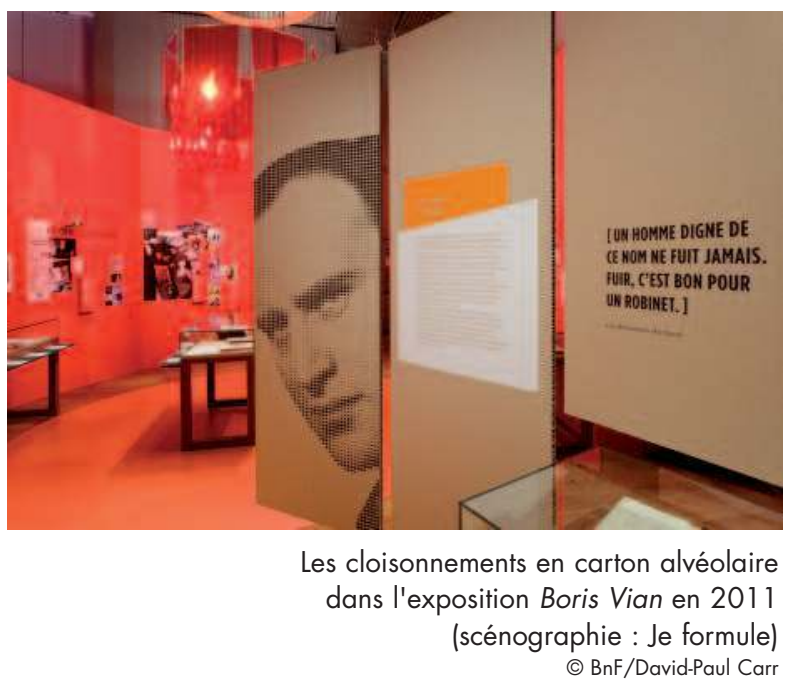

Livingboard ou Agepan, pour des raisons de coût. Depuis 2008 , l'exigence de l'écolabel européen à minima pour les peintures, a été choisi sans qu'il y ait une quelconque incidence financière.

La réutilisation est favorisée. Sur le site de Richelieu, des cimaises mobiles conçues par l'agence NC pour l'exposition Atget (2007), ont servi pour les quatre expositions qui ont suivi. Le parc permanent de vitrines est entretenu, avec toutefois la limite de nos capacités de stockage et de manipulation. La liste de tous nos mobiliers est donnée à chaque concours. Il existe un parc important de capots de verre préférés au plexiglas en raison de sa durabilité et de sa composition. Par contre, les manipulateurs de ces vitrines subissent quotidiennement la contrainte du poids du verre lors des montages!

Le dialogue avec les scénographes, qui, eux aussi, sont de plus en plus engagés dans cette démarche, est très fécond. En effet, ils jouent un rôle de veille important sur les matériaux et nous sommes à l'écoute de leurs propositions innovantes.

\section{Le graphisme ef les travaux graphiques, un domaine en évolution}

Lors des concours, il est fait appel à un binôme scénographe-graphiste. En tant que concepteurs, reviennent aux graphistes certaines orientations déterminantes, en particulier sur le choix des supports, la limitation des quantités, l'évitement des aplats de couleurs, et des couleurs métalliques aux produits nocifs.

Pour répondre à nos appels d'offre de travaux graphiques, les candidats doivent convaincre de leur 
expertise. Depuis 2007, nos interlocuteurs ont obtenu ou sont en voie d'obtenir le label Imprim'vert, ce qui garantit non pas l'utilisation de supports écologiques, mais un fonctionnement responsable des entreprises. Avec ces entreprises, des solutions sont trouvées : cartels PVC remplacés par le PET (polyester issu de matières recyclées), bannières Jet tex dans la gamme Evergreen, encres à éco-solvants. Quelquefois, comme dans l'exposition Boris Vian, nous ne renonçons pas au rendu de la sérigraphie, qui même sans plomb dans les encres, nécessite toujours du solvant pour le nettoyage des cadres.

Depuis 2008, le recyclage et la réutilisation des bannières sont favorisés. La société Bilum récupère ainsi certaines d'entre elles pour fabriquer des articles de bagagerie, chapeaux... en faisant travailler des ESAT (Établissement et services d'aide par le travail). Cette initiative oblige à intégrer une mention concernant cette réutilisation dans les contrats des graphistes, afin d'éviter les problèmes de droits.

Du point de vue administratif, tous les marchés, qu'ils concernent la conception ou les travaux, intègrent les critères du développement durable. Si les exigences sont inscrites dans le cahier des charges, lors de l'analyse, elles sont prises en compte de fait : c'est le cas pour les travaux d'aménagement. Pour les marchés de travaux graphiques, le critère de prise en compte du développement durable pèse pour $20 \%$ dans l'analyse des offres.

\section{L'éclairage avec la Led, une importante réduction de la consommation}

L'équipe technique avait un fonctionnement vertueux depuis longtemps : les salles d'expositions sont

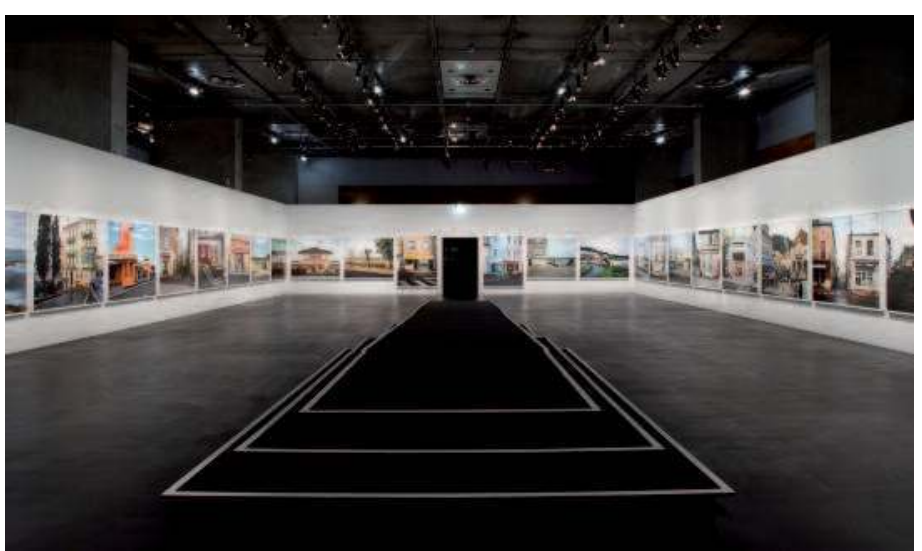

Un plancher réutilisable pour l'exposition Depardon en 2009 (scénographie : Olivia Berton, Julia Kravtsova) ๑) BnF/Emmanuel Nguyen Ngoc systématiquement hors tension pendant la période de fermeture au public, le parc de projecteurs est réutilisé grâce à un entretien rigoureux et des opérations de maintenance régulières. Une fois par semaine, la récupération d'ampoules est organisée dans la BnF. Toutes les ampoules sont basse consommation et un système d'allumage progressif est en place pour éviter leur usure.

Toutefois, l'équipement de certaines salles en Led (Light Emitting Diode) a progressé. La consommation de ces ampoules est 7 fois moins importante. Elles ont une durée de vie de 5000 heures. Cependant, à ce jour, il n'y a pas de visibilité à long terme sur la durée de l'électronique associée à ce système d'éclairage à Led. Un des avantages de ce système, est qu'il supprime toutes les tâches de relampage, ce qui représente une économie sur le long terme qui compense l'investissement, encore important (le système Led revient environ six fois plus cher qu'une ampoule à incandescence classique).

Dès 2008, 80 projecteurs à Led ont été achetés pour tester ceux-ci, notamment sur l'espace permanent de présentation des Globes de Coronelli ; par la suite, la galerie des donateurs a été équipée. Enfin, en 2010, la galerie de photographie sur le site Richelieu a été entièrement équipée grâce à un mécénat en industrie, soit 200 projecteurs fournis par Procédés Hallier.

Ainsi les trésors photographiques du XIXe de l'exposition Les primitifs de la photographie (2010), ont pu benéficier pour la première fois de cet éclairage. Point important pour la conservation des œuvres, il n'y a plus d'émanation de chaleur avec la Led. Il faut signaler que la perception visuelle des espaces d'exposition éclairés par Led est différente : ceux-ci paraissent plus lumineux, le blanc étant plus présent. Cela implique un changement d'habitude à la fois pour les usagers, mais également pour ceux qui mettent en œuvre l'éclairage et le choix des couleurs de cimaises.

\section{Les actions en lien avec d'autres secteurs d'activités de la BnF}

Nous venons de lister les actions directement gérées par le service des expositions, mais un certain nombre d'éléments liés à la gestion des expositions temporaires concernent également d'autres secteurs de la Bibliothèque.

L'encadrement des œuvres exposées est fait soit par des prestataires extérieurs, soit par des restaurateurs de la BnF. Les pratiques de récupération font partie 
de la culture professionnelle des ateliers de conservation de la BnF. Par exemple, $40 \%$ des passe-partout sont réutilisés. Les cadres faits à partir de bois labellisés sont réutilisés le plus possible, mais nos collègues sont dépendants de la capacité de stockage des ateliers.

Les équipements informatiques sont labellisés Energy star, et la redistribution à des associations des postes informatiques usagés a été mise en place par la direction informatique de l'établissement.

Une des recommandations d'Atémia était de compenser les transports liés aux déplacements des œuvres. En fait, depuis 2010, la BnF a adopté la compensation carbone des transports liés aux déplacements en avion de ses agents, à l'emprunt d'œuvres pour ses expositions, et enfin au prêt de documents. Dans ce dernier cas, il est proposé aux emprunteurs, sur la base du volontariat, de compenser les dégagements de $\mathrm{CO}_{2}$ induits par le transport auprès de la société Climat mundi (4) avec qui la $\mathrm{BnF}$ a un contrat. Cette opération est relativement symbolique pour les emprunts d'œuvres qu'exigent les expositions, puisque nous essayons de limiter au maximum les transports aériens.

Enfin, il existe encore des difficultés à mettre en œuvre un traitement vraiment responsable des déchets. L'étude avait révélé que pour 2007, 16 tonnes de déchets étaient évacuées pour les expositions. C'était avant que nous ne fassions plus léger! Il n'y a pas de tri organisé au sortir des expositions, car l'évacuation se fait globalement par le titulaire du marché BnF et son contrat ne garantit pas le tri. La récupération des

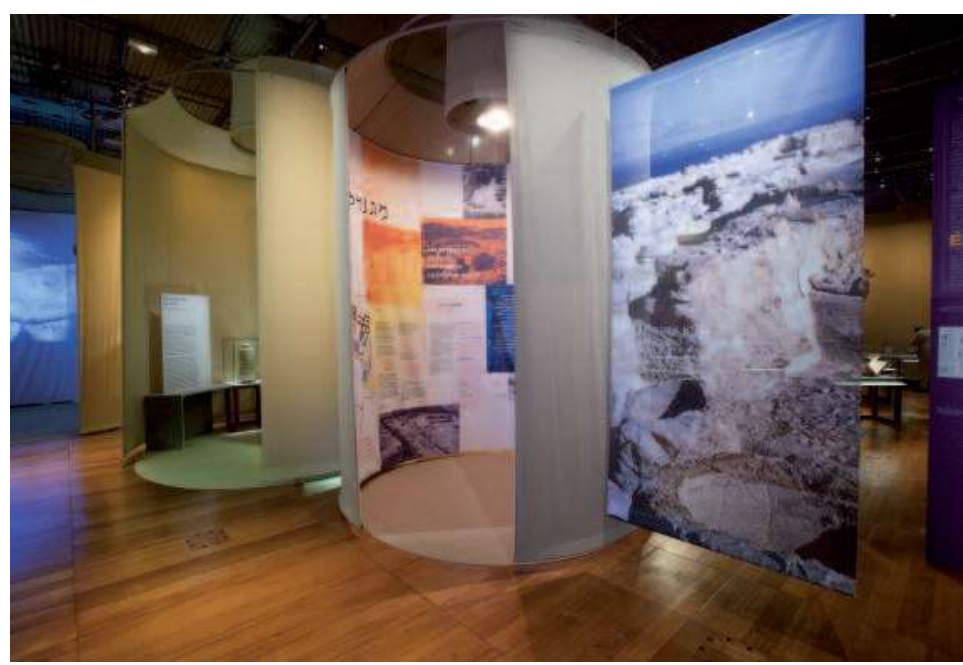

L'exposition Qumran, le secret des manuscrits de la Mer Morte en 2009 (scénographie : MAW) ( ) BnF/David-Paul Carr bannières de signalétique par Bilum est un premier pas ; un contact a été pris avec la Réserve des Arts ${ }^{(5)}$, une association parisienne qui vend à bas prix aux artistes des matériaux issus d'expositions ou de spectacles. Toutefois, nos déchets un peu trop volumineux ne les intéressent pas directement, car pour eux, comme pour nous, le problème récurrent est le stockage de transit. En effet, l'organisation de la récupération des déchets ou des consommables est consommatrice d'espace, de temps, et de manutention. À ce jour, cette question pourtant essentielle des déchets n'a pas encore trouvé de solution satisfaisante.

\section{Une mise en œuvre collective au sein du service des expositions}

Les résultats de l'étude ont été transmis à tous les agents dès 2008. Un référent « développement durable » a été désigné dans le service et en 2010, tous les chargés de projet ont pu bénéficier d'une journée bilan-formation. À ce jour, chacun s'est saisi de cette réflexion. Les réticences du début ont disparu, les craintes sur les surcoûts occasionnés par une telle politique ne sont plus valides au vu de l'expérience de ces trois années. Dorénavant, des points réguliers sur les projets des uns et des autres, les problèmes rencontrés, les solutions trouvées sont faits en réunion de service. Nous sommes passés désormais à l'étape suivante, celle de l'évaluation. Depuis 2011, chaque chargé de projet évalue ses expositions avec un questionnaire standard auto-administré. Ce questionnaire sert surtout de guide méthodologique et permet de voir si dans l'ensemble nous progressons. Il attribue à chaque partie du projet (conception, fabrication, utilisation, transport, fin de vie) un certain nombre de points.

En phase conception et fabrication, la plupart des projets ont des «bonnes notes »; pour la fin de vie, la marge de progression est encore importante.

\section{Le défi suivant : conforter l'engagement éco-responsable sur la durée}

Si les réflexes de base sont acquis au sein de l'équipe, si chacun s'est approprié la démarche, la dynamique collective est à préserver : rien n'est acquis, tout récemment le parc de vitrines s'est trouvé menacé, non en tant que tel bien sûr, mais parce que l'espace où il était stocké devait être affecté à un autre usage. Autre exemple, la conception de certains projets d'exposition obéissant à des contraintes artistiques fortes - comme l'exposition Richard Prince, American Prayer - nous oblige parfois à quelques entorses à nos propres recommandations. 


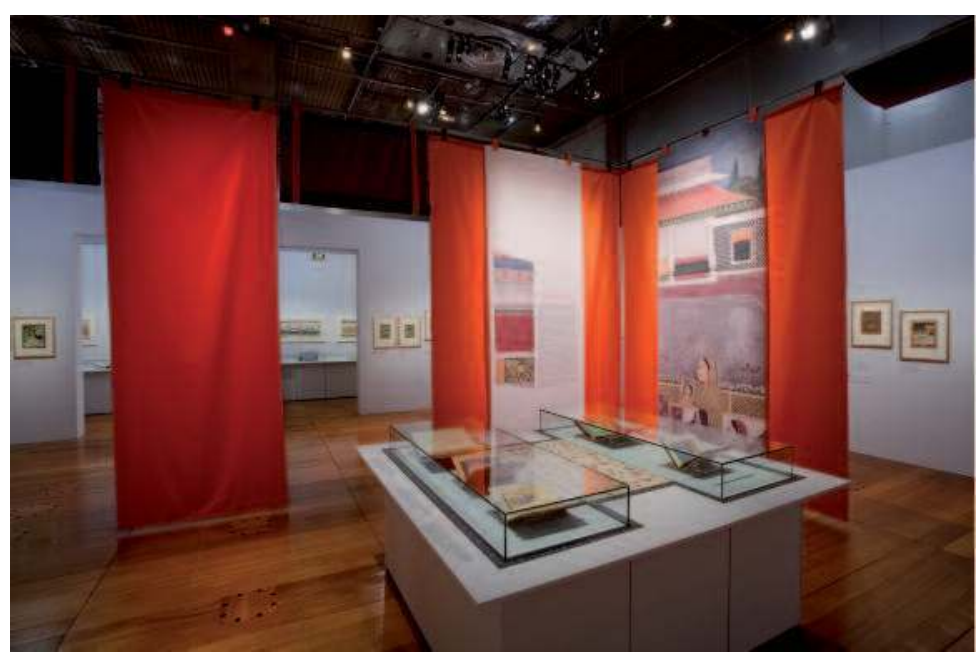

L'exposition Miniatures indiennes en 2009 (scénographie : Véronique Dollfus) (c) BnF/David-Paul Carr

Par ailleurs, certains freins sont toujours là et dépassent les actions de notre service : par exemple, les procédures administratives et financières sont encore à adapter ; avec le cadre juridique de la propriété d'État, il est difficile de favoriser légalement la réutilisation des éléments d'exposition par des organismes. Le code des marchés publics ne tient pas compte de la proximité géographique des fournisseurs. Une gestion durable des expositions temporaires, avec réutilisation et recyclage des matériaux nécessite des espaces de stockage, de la main d'œuvre ; pour l'instant, nous agissons au mieux de nos possibilités dans un contexte budgétaire contraint et avec une programmation d'expositions copieuse.
Le choix d'une démarche progressive et partagée a été fait : nos expositions sont, sauf exceptions, plus économes, plus respectueuses de l'environnement et tout aussi réjouissantes. Nos partenaires, scénographes, graphistes et fournisseurs sont eux aussi engagés dans cette démarche, et c'est ensemble que nous progressons. Mutualiser les expériences, échanger avec d'autres concepteurs d'expositions, responsables de musées, voici ce qui pourra nous aider à être encore un peu plus « exemplaires » et garder le souffle de nos convictions.

\section{Notes}

(1) Bibliothèque nationale de France : www.bnf.fr. Présentation des expositions, expositions virtuelles.

(2) Atémia : www.atemia

(3) Disponible sur simple demande auprès des auteurs

(4) Climat mundi : www.climatmundi.fr

(5) La Réserve des Arts. Paris, 20e arrondissement : wwwllareservedesarts.org 